BULL. AUSTRAL. MATH. SOC.

06A20, 46L05, 54D10,54D99

VOL. $21(1980), 265-279$.

\title{
A NOTE ON BAIRE SPACES AND CONTINUOUS LATTICES
}

\author{
KarL H. HOFMANN
}

\begin{abstract}
We prove a Baire category theorem for continuous lattices and derive category theorems for non-Hausdorff spaces which imply a category theorem of Isbell's and have applications to the spectral theory of $C^{*}$-algebras. The same lattice theoretical methods yield a proof of de Groot's category theorem for regular subcompact spaces.
\end{abstract}

\section{Background and main results}

A topological space is a Baire space if any countable intersection of dense open sets is dense. The classical result on such spaces is the category theorem of Baire.

THEOREM A (Baire). A space is a Baire space if it is locally compact Hausdorff or completely metrizable.

This theorem is eminently useful in analysis and topological algebra; one need only recall the open mapping theorems for topological groups and topological vector spaces (see, for example, [1], p. 120) or of theorems in which joint continuity of a two variable function is deduced from separate continuity (see, for example, [1], p. 12l, [13], [14], [15]). Basic properties of Baire spaces and their applications are developed in standard texts such as Bourbaki's [1]. The Baire category theorem is in reality two theorems in one; it was de Groot who succeeded in finding a common generalisation of the two. He formulated the following condition [7].

Received 25 October 1979. 
DEFINITION B (de Groot). A space is called subcompact if there is a basis $B$ for the topology such that every filterbasis $F$ on $B$ has a non-empty intersection provided it is regular, that is satisfies the condition that $V \in F$ implies the existence of a $U \in F$ with $\bar{U} \subset V$.

He then proves:

THEOREM C (de Groot). Every subcompact regular space is a Baire space.

All locally compact Hausdorff spaces are subcompact (take for $B$ the set of all relatively compact open sets) and all completely metrizable spaces are subcompact (take for $B$ the set of open balls of radius $1 / n$ with $n=1,2, \ldots$ ). Thus de Groot's theorem unifies Baire's.

In fact, de Groot establishes more general category theorems in which higher cardinals replace countability [7]; a gap in his proof was only recently closed by Fleischer [4]. In the paper before us higher cardinals will not be the issue.

Another category theorem arising in functional analysis is perhaps less known; it is due to Choquet and is published in [3].

THEOREM D (Choquet). The space of extreme points of a compact convex set in a locally convex topological vector space is a Baire space.

If one now considers the dual of a $C^{*}$-algebra $A$ with the weak topology and therein the set $P(A)$ of pure states, then Choquet's theorem shows that $P(A)$ is a Baire space. One knows that there is a continuous and open map from $P(A)$ onto the primitive ideal spectrum Prim $A$ of $A$ (see [3], p. 68), and so one obtains

THEOREM E (Dixmier). The primitive ideal space Prim $A$ of a $C^{*}$ algebra $A$ is a Baire space.

Here we encounter spaces which are no longer Hausdorff. Spectra of rings and algebras satisfy the axiom $T_{0}$, but generally no other separation axiom. It is therefore important to understand category theorems of the Baire type for $T_{0}$-spaces. It is true that the primitive ideal space of a $C^{*}$-algebra is locally quasicompact; but one should not believe that locally quasicompact spaces are Baire spaces in the absence of Hausdorff separation. Some very simple examples will illustrate this 
point. First recall, that a space is locally quasicompact, if every point has a neighborhood basis of quasicompact neighborhoods.

EXAMPLE 1.1. Let $X$ be a well-ordered set. Then the collection of all $U_{x}=\{y \in X: x \leq y\}$ together with $X$ and $\emptyset$ forms a topology. Every open set is the smallest neighborhood of its minimum (or else is empty), hence is quasicompact. Further, every non-empty open set is dense. Special cases:

(1) Let $X=\mathbb{N}$ be the set of natural numbers in their natural order. Then $X$ is a locally quasicompact $T_{0}$-space which is not a Baire space, since the intersection of the dense open sets $U_{n}, n \in \mathbb{N}$, is empty.

(2) Let $X=\Omega$ be the set of all countable ordinals in their natural order. Then $X$ is a locally quasicompact $T_{0}$ Baire space.

Before we further discuss non-separated spaces we need to introduce a property, which arises very naturally in algebraic geometry and in the spectral theory of rings and algebras and which partially makes up for the loss of separation. Firstly, a closed subset $A$ of a space $X$ is called irreducible, if it is not the union of two closed proper subsets. If $X$ is the space of Example l.I (1), then $A=X$ is irreducible. Any singleton closure is irreducible. Secondly, a space is called sober, if each nonvoid closed irreducible subset has a unique dense point. Notice that neither Example 1.1 (1) nor Example 1.1 (2) is a sober space, since $X$ has no maximum, hence no dense point. Because of the uniqueness requirement in the definition of a sober space all sober spaces are $T_{0}$-spaces.

I am not sure whether it is well-known that one has a Baire category theorem for locally quasicompact sober spaces. In any case one finds such a result in a paper of Isbell's [12].

THEOREM F (Isbell). Every Locally quasicompact sober space is a Baire space.

The key is lattice theoretical. It has been recognized several times that it is useful to consider the lattice theoretical background of the Baire category theorem (see, for example, Fleischer and Reyes [5]). Isbel I obtained his category theorem pursuing "pointless topology"; that is, 
the study of complete lattices satisfying the infinite distributive law $x \wedge \sup X=\sup (x \wedge X)$ for any subset $X$ and any element $x$ of the lattice. The lattice of open sets of any space is of this type. These lattices have been given many different names; such as complete Brouwerien lattices, local lattices, locales, frames, complete Heyting algebras. . Isbell makes the point that in general one does not expect a Baire category theorem for complete Heyting algebras ([12], p. 334). We concentrate here on a different type of complete lattice which in general need not even be distributive. Indeed, much attention has been given recently to continuous lattices, so called by Scott in his construction of set theoretical models of the lambda calculus [16] and in his mathematical foundation of computation. In topology and topological algebra, continuous lattices occur frequently as I pointed out in my survey article [9]; a general treatment of continuous lattices is about to become available [6].

Among the numerous characterisations of continuous lattices, the one using an auxiliary relation is still the most convenient and straightforward for the definition:

DEFINITION 1.2. (1) Let $L$ be a complete lattice. Then we write $x \ll y$ for two elements whenever for any directed set $D$ with $y \leq \sup D$ we find a $d \in D$ with $x \leq d$.

(2) A continuous lattice is a complete lattice in which every element $y$ satisfies the relation $y=\sup \{x: x<y\} \quad$ ([6], I-I.I and I-1.7).

We read the relation $x \ll y$ as " $x$ is way below $y$ ". This relation is readily seen to be transitive and stronger than the relation $\leq$; in general it is not reflexive. Because of Definition 1.2 (2), the way below relation holds fairly often in a continuous lattice. For more technical information turn to [6], Chapter I, Section 1. We note that for a topological space $X$ the lattice $O(X)$ of open sets is a complete lattice (and indeed a complete Heyting algebra). If $U, V \in O(X)$ and $Q$ is a quasicompact set with $U \subset Q \subset V$, then $U \ll V$. Conversely, if $X$ is locally quasicompact, then $U \ll V$ implies the existence of a quasicompact set $Q$ with $U \subset Q \subset V$, and condition (2) of Definition 1.2 is satisfied. Thus $O(X)$ is a continuous lattice whenever $X$ is a locally quasicompact space. Lawson and I showed in [11], that for sober spaces this condition is also sufficient. We also proved that there are second 
countable $T_{0}$-spaces $X$ for which $O(X)$ is a continuous lattice, but in which every quasicompact set has empty interior. Nevertheless, spaces for which $O(X)$ is a continuous lattice behave in many respects like locally compact spaces. This justifies the following definition [17].

DEFINITION 1.3 (Ward). A space $X$ is quasi-locally compact if $O(X)$ is a continuous lattice.

Recall: a locally quasicompact space is quasi-locally compact but not conversely. For sober spaces, the two concepts agree. Quasi-locally compact spaces have also been called semi-locally bounded (|sbel|), core compact (Hofmann and Lawson), $C L$-spaces (Hofmann). For equivalent characterisations of these spaces see, for example, [11].

An element $p$ in a lattice is called irreducible, if the relation $p=x y$ implies $p=x$ or $p=y$. The set of irreducible elements $p<1$ in a lattice $L$ will be denoted $\operatorname{IRR}_{1} L$. The irreducible closed sets in a space $X$ have now a clear-cut lattice theoretical meaning: a closed subset $A$ of $X$ is irreducible if and only if $X \backslash A \in \operatorname{IRR}_{1} O(X)$. If $X$ is sober, then the function $x \mapsto X \backslash\{\bar{x}\}: X \rightarrow \operatorname{IRR}_{1} O(X)$ is a bijection. Under these circumstances, if $P=X \backslash\{\bar{x}\} \in \operatorname{IRR}_{2} O(X)$ and $U \in O(X)$ we have $x \in U$ if and only if $U \notin P$; the point is that the second relation is formulated entirely within the lattice $O(X)$. This allows us to interpret in an arbitrary lattice $L$ the irreducibles as "points" and to interpret the relation $u \neq p$ between an arbitrary element $u \in L$ and an irredicuble $p$ as "the point $p$ is an element of $u$ ". With this in mind we begin to formulate the Baire property in lattice theoretical terms. Of course, we also need to express in lattice theoretical terms what it means that an open set is dense in a space. This is easy.

DEFINITION 1.4. An element $d$ in a lattice $L$ with zero is dense, if the relation $0 \neq x$ in $L$ always implies $x \wedge d \neq 0$ for all $x$ ( $c f$. [5]).

We are now ready for the principal definition.

MAIN DEFINITION 1.5. A complete lattice $L$ is called a Baire lattice, if for every countable collection $N \subset L$ of dense elements and for each non-zero element $u \in L$ there is an irreducible element $p$ such 
that $d \wedge u \neq p$ for all $d \in N$.

Notice that if $L=O(X)$ for a sober space $X$, this says precisely that $X$ is a Baire space.

Now we are ready for the main theorem.

MAIN THEOREM 1.6. Every continuous lattice is a Baire lattice.

Before we proceed to the proof and a discussion we derive the relevant topological corollary. An immediate translation yields

BAIRE CATEGORY THEOREM FOR QUASI-LOCALLY COMPACT SPACES 1.7. Let $X$ be a quasi-locally compact space. Then for each countable family $D_{n}$, $n \in \mathbb{N}$ of dense open subsets and for each non-empty open set $U$ there is an irreducible closed set $A$ in $X$ such that $A \cap U \cap D_{n} \neq \emptyset$ for alz $n \in \mathbb{N}$.

This applies in particular to locally quasicompact spaces. Isbell's category theorem (Theorem $F$ ) is an immediate corollary of Theorem 1.7. For an illustration of Theorem 1.7 one might wish to consider Example 1.1 (1). Notice that Theorem 1.6 is more general than Theorem 1.7 since it does not require distributivity of the lattice. We will in fact provide a lattice theoretical theorem which will both imply our main theorem and de Groot's Theorem C. It should be interesting to observe how regularity intercedes in the latter.

We saw that for quasi-locally compact spaces, sobriety implies the Baire space property. Example 1.1 (2) shows that the converse fails. How closely are the two concepts related? Before we give some answers, we recall the following facts. For any topological space $X$ there is an open continuous map $s_{X}: X \rightarrow \bar{X}$ into a sober space such that

$U \mapsto s_{X}^{-1}(U): O(\check{X}) \rightarrow O(X)$ is a lattice isomorphism. The map is natural and universal (that is, every continuous function $X \rightarrow Y$ into a sober space factors through $s_{X}$ ). If $X$ is a $T_{0}$-space, then $s_{X}$ is an embedding (see [8], [11]). The space $\check{X}$ is called the sobrification of $X$. For the following proposition recall that not every closed subspace of a Baire space is in general a Baire space.

PROPOSITION 1. Let $X$ be a quasi-locally compact $T_{0}$-space such 
that its sobrification $\bar{X}$ is first countable. Then the following conditions are equivalent:

(1) $X$ is sober;

(2) all closed subspaces of $X$ are Baire spaces;

(3) all closed irreducible subspaces of $X$ are Baire spaces.

The first axiom of countability of $\bar{X}$ does not translate in a simple fashion into a property of $X$. But since $O(\bar{X}) \cong O(X)$, we know that $\check{X}$ is second countable if and only if $X$ is second countable, and second countability implies first countability. Hence we have the corollary:

COROLLARY 1.9. Let $X$ be a second countable quasi-locally compact $T_{0}$-space. Then the above conditions (1), (2) and (3) (of Proposition 1.8) are equivalent.

Taking Corollary 1.9 together with Dixmier's category theorem (Theorem E) we deduce that $\operatorname{Prim} A$ is sober for any separable $C^{*}$-algebra $A$, since the primitive ideal space of a separable $C^{*}$-algebra is second countable. The lattice of closed two sided ideals of $A$ is isomorphic to $O(\operatorname{Prim} A)$. Hence we know that $\operatorname{Prim} A$ is sober if and only if every closed prime ideal is primitive. Thus we obtain the following corollary, for which a different proof is given in [3], p. 79.

COROLLARY 1.10. In a separable $C^{*}$-algebra every closed two-sided prime ideal is the kernel of an irreducible representation.

In the absence of more precise information, let us denote by PRIM $A$ the space of closed two sided prime ideals of a $C^{*}$-algebra $A$. Then PRIM $A=(\operatorname{Prim} A)^{-}$, and as a consequence of the fact that the spectrum of a continuous lattice is locally quasicompact and sober and of our results above we may record

PROPOSITION 1.11. The space PRIM $A$ is a locally quasicompact sober $T_{0}$ Baire space for any $C^{*}$-algebra $A$.

I wish to acknowledge a seminar discussion with Horst Leptin who asked me whether there was an access to the Baire space property of primitive ideal spectra of $C^{*}$-algebras through continuous lattices. I still cannot answer this affirmatively, but his question led me to the main theorem. 


\section{More lattice theory: proofs}

We begin with some notation. Let $L$ be a lattice. Instead of $x \wedge y$ we will write $x y$, and for a subset $x \subset L$ we set

$$
\downarrow X=\{y \in L: y \leq x \text { for some } x \in X\}
$$

and

$$
X=\{y \in L: x \leq y \text { for some } x \in X\} .
$$

We abbreviate $\downarrow\{x\}$ by $\downarrow x$ and $\uparrow\{x\}$ by $\nmid x$. A set $X \subset L$ is called an upper set if $\uparrow X=X$. A set $F \subset L$ is a filter if it is an upper set and satisfies $F F \subset F$.

Much of continuous lattice theory is based on the auxiliary relation $\ll$ which we introduced in Definition 1.2 (1). It is useful, however, to deal with auxiliary relations on a formal basis (see [6], I-1.9).

DEFINITION 2.1. A binary relation $\prec$ on a lattice will be called an extra order, if it satisfies the following conditions for all $u, x, y, z \in L$ :

(1) $x-y$ implies $x \leq y$;

(2) $u \leq x \prec y \leq z$ implies $u \prec z$;

(3) $0<x$.

An extra order is said to be regular, if, in addition, it also satisfies

(4) $z \neq 0$ implies there is a $v \neq 0$ with $v<z$.

Some extra orders satisfy the following condition which is stronger and is called the interpolation property:

(INT) $(x<z$ and $x \neq z)$ implies there is a $v \neq x$ with $x<v<z$.

REMARK 2.2. (1) The way-below relation $\ll$ on a continuous lattice is an extra order with the interpolation property; in particular, it is regular (see [6], I-I for these facts and further details).

(2) Let $L=O(X)$ for a topological space $X$. Suppose that $B$ is a basis for the topology. For $U, V \in O(X)$ we write $U \prec V$ if there is a $W \in B$ such that $U \subset \bar{W} \subset V$. Then $\prec$ is an extra order which is certainly regular if $X$ is a regular space. 
DEFINITION 2.3. If $L$ is a lattice with an extra order $\prec$, we say that an upper set $U$ is open in $(L, \prec)$, if for every $v \in U$ there is a $u \in U$ with $u \prec v$. In an arbitrary complete lattice $L$ we say that an upper set $U$ is open (without specification), if for every $v \in U$ there is a $u \in U$ with $u \ll v$ (that is if $U$ is open in $(L, \ll)$ ) (see [6], I-3.1).

We are now ready for the key lemma, whose proof for continuous lattices is Keimel's streamlined version of my original proof.

LEMMA 2.4. Let $L$ be a lattice with an extra order $\prec$. Suppose that $V$ is an open upper set in $(L,-)$. If $N$ is any countable subset of $L$ with $V N \subset V$, then for each $v \in V$ there is an open filter $U$ in $(L, \prec)$ with $v N \subset U \subset V$.

Proof. The set $G=\{x \in L: V x \subset V\}$ is a filter containing $N=\left\{x_{n}: n=1,2, \ldots\right\}$. We define $a_{n}=x_{1} \ldots x_{n}$ and note $a_{n} \in G$ for all $n=1,2, \ldots$. We select inductively a sequence $b_{n} \in V$, $n=0,1, \ldots$ as follows: set $b_{0}=v$ and suppose that $b_{0}, \ldots, b_{n} \in V$ have been selected. Since $a_{n+1} \in G$ we have $b_{n} a_{n+1} \in V$. Since $V$ is open in $(L, \prec)$, we find an element $b_{n+1} \in V$ with $b_{n+1} \prec b_{n} a_{n+1}$. Since $b_{n} a_{n+1} \leq b_{n}$, we can apply Definition 2.1 (2) to obtain $b_{n+1} \prec b_{n}$. Similarly, from $b_{n} a_{n+1} \leq a_{n+1}$ we obtain $b_{n+1} \prec a_{n+1}$ via Definition 2.1. (2), which implies $b_{n+1} \leq a_{n+1}$ by Definition 2.1 (1). We now set $U=U\left\{\uparrow b_{n}: n=0,1, \ldots\right\}$. Since $b_{n} \in V$ for all $n$, clearly $U \subset V$. In particular, $v=b_{0} \in U$ and $a_{n} \in \uparrow b_{n} \subset U$ for all $n$, and so $x_{n} \in U$ for all $n$. If $x, y \in U$, then there is an $n$ with $b_{n} \leq x, y$, and thus $x y \in \uparrow b_{n} \subset U$. Hence $U$ is a filter. Therefore, $v a_{n} \in U$ for all $n$. If $u \in U$, then there is an $n$ with $b_{n+1}<b_{n} \leq u$, and thus $b_{n+1} \prec u$ by Definition 2.1 (2). Hence $U$ is open in $(L, \prec)$.

DEFINITION 2.5. A pair $(L, \prec)$ of a lattice with an extra order is called a de Groot lattice, if for every open filter $U$ in $(L, \prec)$ there is an irreducible element $p \in L \backslash U$. We say that $(L, \prec)$ is regular if $\prec$ is regular. 
LEMMA 2.6 ([6], I-3.4 and 3.6). Let $L$ be a complete lattice and $U$ an open filter. Then for any $x \notin U$ there is an irreducible element $p \notin U$ with $x \leq p$. In particular, $(L, \ll)$ is a de Groot lattice.

The proof is easy: one uses the openness of $U$ and the definition of $\ll$ to show that $\uparrow x \backslash U$ is inductive and picks a maximal element $p$ in $\uparrow x \backslash U$.

PROPOSITION 2.7. Let $L$ be a continuous lattice and $V$ an open upper set. If $N$ is a countable set with $V N \subset V$, then for every $v \in V$ and every $x \notin V$ there is an irreducible element $p \geq x$ with $p \nmid \downarrow v$.

Proof. Let $U$ be the open filter constructed in Lemma 2.4 with $\ll$ in place of $\prec$. Let $p$ be as in Lemma 2.6.

This proposition generalizes some well known facts on continuous lattices.

COROLLARY 2.8. Let $L$ be a continuous lattice. Then

(1) if $y \neq x$, then there is an irreducible element $p$ with $x \leq p$ and $y \neq p$;

(2) if $y \neq x$, then there is an open filter $U$ with $y \in U$ and $x \notin U$.

Proof. Conclusion (2) is immediate from Lemma 2.4 with $N=\{1\}$ and $\ll$ in place of $<$, and conclusion (1) follows from (2) and Lemma 2.6, or else directly from Proposition 2.7 with $N=\{1\}$.

Conclusion ( 1 ) is equivalent to the statement $x=\inf \left(\uparrow x \cap \operatorname{IRR}_{1} L\right)$ for all $x \in L$.

We return for a moment to another class of de Groot lattices, which in fact suggested the name.

PROPOSITION 2.9. If $X$ is a subcompact space and $B$ the basis given in de Groot's Definition $B$, and if we define $U \prec V$ in $O(X)$ if and only if there is a $W \in B$ such that $U \subset \bar{W} \subset V$, then $(O(X),-)$ is $a$ de Groot lattice which is regular if $X$ is a regular space (see Remark $2.2(2)$ and Definition 2.5).

Proof. Let $u$ be an open filter in $(O(X),-<)$. Then $u \cap B$ is a 
filter basis on $B$ such as was demanded in Definition $B$. It therefore contains a point in its intersection by subcompactness. If $x$ is this point, then $X \backslash\{x\}^{-}$is an irreducible element of $O(X)$ which is not contained in $U$.

Now we have two instances of regular de Groot lattices: continuous lattices (Remark 2.2, Lemma 2.6) and topologies of subcompact regular spaces (Proposition 2.9). At this point we formulate a lattice theoretical generalisation of de Groot's Theorem $C$.

THEOREM 2.10. Let $L$ be a complete lattice with an extra order $<$ such that $(L,-)$ is a regular de Groot lattice. Then $L$ is a Baire Zattice.

Proof. Let $N$ be a countable set of dense elements. This means that $0 \neq x$ and $n \in N$ always implies $0 \neq x n$. Let $v \neq 0$ be arbitrary in $L$. We set $V=L \backslash\{0\}$. By Definition 2.I (4), the upper set $V$ is open in $(L, \prec)$, and by what we just saw, $V N \subset V$. By Lemma 2.4 there is an open filter $U$ in $(L, \prec)$ with $v N \subset U \subset V=L \backslash\{0\}$. Since $(L, \prec)$ is a de Groot lattice, there is an irreducible element $p \in L \backslash U$. Hence $p \notin \uparrow v N$ which we had to show (Definition 1.5).

We can now readily prove some of the main results of Section 1.

Proof of the Main Theorem 1.6. For a continuous lattice, « is regular (Remark 2.2 (1)); so by Lemma 2.6 we know that $(L, \ll<$ is a regular de Groot lattice. Hence $L$ is a Baire lattice by Theorem 2.10.

Proof of de Groot's Theorem C. Let $X$ be a regular $T_{0}$-space. Then it is Hausdorff, hence sober. Now $X$ is a Baire space if and only if $O(X)$ is a Baire lattice. But if $X$ is regular, then the extra order of Remark 2.2 (2) and Proposition 2.9 is regular. Hence $(O(X), \prec)$ is a regular de Groot lattice by Proposition 2.9. Thus $O(X)$ is a Baire lattice by Theorem 2.10. The assertion thus is proved for $T_{0}$-spaces. Since an arbitrary space is a Baire space if and only if its universal $T_{0}$-quotient (in which points $x$ and $y$ are identified if and only if $\{x\}^{-}=\{y\}^{-}$, is a Baire space, the theorem is proved.

We now proceed to prove Proposition 1.8. Some further notation is needed. On a complete lattice we introduce a $T_{0}$-topology $\omega(L)$, called 
the Zower topology, by taking the sets $L \backslash \uparrow x$ as subbasic. A set $X \subset L$ is called order generating, if $x=\inf (\uparrow x \cap X)$ for all $x \in L$. If $X \subset \operatorname{IRR}_{1} L$, then we call the topology induced on $X$ by $\omega(L)$ the hulZkernel topology. If $L$ is distributive and thus $\operatorname{IRR}_{1} L$ is the set of prime elements below 1 , this is the traditional concept.

LEMMA 2.11. Let $L$ be a continwous lattice with $0 \in \operatorname{IRR}_{1} L$. If $X \subset \operatorname{IRR}_{1} L$ is an order generating set with $0 \notin X$, and if $\operatorname{IRR}_{1} L$ is first countable in 0 , then $X$ is not a Baire space.

Proof. Step 1. For $x \neq 0$, the closed set $\uparrow x \cap X$ is nowhere dense: indeed, if it is, then it contains a basic non-empty open set $U=X \backslash\left(\uparrow a_{1} \cup \ldots \cup \uparrow a_{n}\right)$; that is, $X \subset \uparrow x \cup \uparrow a_{1} \cup \ldots \cup \uparrow a_{n} \cdot$ In particular, $X \subset \uparrow x a_{1} \cdots a_{n}$, and thus $x a_{1} \cdots a_{n} \leq$ inf $X=0$, since $X$ is order generating. But since $U \neq \emptyset$, neither $x$ nor any of the $a_{m}$ is 0 , and this is a contradiction to the irreducibility of 0 .

Step 2. Set $y=I R R_{1} L$. Basic $\omega(L)$-neighborhoods of 0 in $y$ are of the form $Y \backslash F$ with a finite set $F$ not containing 0 . Since 0 is irreducible, $0 \neq$ inf $F$; hence 0 has in fact a basis of $\omega(L)$ neighborhoods of the form $Y \backslash \uparrow v$ with $v \neq 0$. Since 0 has a countable basis of $w(L)$-neighborhoods, there is a sequence $v_{1} \geq v_{2} \geq \ldots>0$ such that the sequence $Y \backslash \uparrow v_{n}$ is a neighborhood basis of 0 . If $x \neq 0$, then there must be a basic neighborhood $Y \backslash \uparrow v_{n}$ which is contained in the neighborhood $L \backslash \uparrow x$. Thus $v_{n} \leq x$. Thus $\left\{v_{n}\right\}$ is cofinal in the filter $L \backslash\{0\}$. This means $L \backslash\{0\}=U \uparrow v_{n}$, and thus $X=U\left(X \backslash \uparrow v_{n}\right)$. Hence $X$ is a countable union of the closed sets $X \backslash \uparrow v_{n}$, which are nowhere dense in $X$ by Step 1 . Hence $X$ is not a Baire space.

PROPOSITION 2.12. Let $L$ be a continuous lattice and $X$ an order generating subspace of $\operatorname{IRR}_{1} L$ in the hull-kernel topology. Suppose that each irreducible element $p$ of $L$ has a countable neighborhood basis in $\uparrow p$ relative to the lower topology. If $p \in \operatorname{IRR}_{1} L \backslash X$, then $\uparrow p \backslash X$ is a closed irreducible subset of $X$ which is not a Baire space. 
Proof. We apply Lemma 2.11 with the continuous lattice $t p$ in place of $L$, with $p$ in place of 0 and with $4 p \cap X$ in place of $X$. It remains to show that $\uparrow p \cap X$ is irreducible in $X$. Suppose then $\uparrow p \cap X=A_{1} \cup A_{2}$ with two closed subsets of $\uparrow p \cap X$. Since every closed subset is an intersection of basic ones we may assume that $A_{1}$ and $A_{2}$ are basic. Then they are of the form $A_{n}=X \backslash \uparrow F_{n}$ with two finite sets $F_{n}, n=1,2$, of $L$. Since $A_{n}=\uparrow p \cap A_{n}$, we have $X \cap \uparrow F_{n} \cap \uparrow p=X \cap \uparrow\left(F_{n} \vee p\right)$, and so we may assume that $F_{n} \subset \uparrow p$, for $n=1,2$. Since $X$ is order generating, we have

$$
p=\inf (\uparrow p \cap X)=\inf \left(A_{1} \cup A_{2}\right) \geq \inf \left(F_{1} \cup F_{2}\right) \geq p \text {. }
$$

Since $p$ is irreducible, this implies $p \in F_{1}$ or $p \in F_{2}$, and this means $\uparrow p \cap X \subset A_{1}$ or $\uparrow p \cap X \subset A_{2}$, and this shows that $\uparrow p \cap X$ is irreducible.

As a corollary we now prove Proposition 1.8. By the results of [11], we may assume that $X$ is an order generating subset of the spectrum $\mathrm{IRR}_{1} L$ of a continuous Heyting algebra $L$, and we know from [11], that $X$ is sober if and only if $X=\mathrm{IRR}_{1} L$. If this condition holds, then $X$ is locally quasicompact sober, and then so is every closed subspace. Thus $X=\operatorname{IRR}_{1} L$ if and only if (1) implies (2) and (2) implies (3) in Proposition 1.8. It remains to show that $X \neq \mathrm{IRR}_{1} L$ implies not (3). Indeed suppose that we have a $p \in \operatorname{IRR}_{1} L \backslash X$. If $\operatorname{IRR}_{1} L$ is first countable, then $\operatorname{IRR}_{1} \uparrow p=\uparrow p \cap \mathrm{IRR}_{1} L$ is first countable with respect to $\omega(\uparrow p)$ in $p$. Since the topology $\omega(L)$ induces on $X$ the given topology and on $\bar{X}=\operatorname{IRR}_{1} L$ the sobrification topology, the hypotheses of Proposition 2.12 are satisfied. Thus Proposition 2.12 provides the existence of an irreducible closed subset of $X$ which is not a Baire space. 


\section{References}

[1] N. Bourbaki, Éléments de Mathêmatique. I: Les structures fondamentales de l'analyse. Livre III: Topologie générale. Chapitre 9: "Utilisation des nombers réels en topologie générale". Deuxième édition revue et augmentée (Hermann, Paris, 1958). See also: Nicolas Bourbaki, Elements of mathematics. General topology, Part 2. Chapter 9: "Use of real numbers in general topology" (Addison-Wesley, Reading, Massachusetts, 1966).

[2] Jacques Dixmier, "Sur les C*-algèbres", BuZZ. Soc. Math. France 88 $(1960), 95-112$.

[3] Jacques Dixmier, Les $C^{*}$-algèbres et leurs représentations, deuxième édition (Cahiers Scientifiques, 29. Gauthier-Villars, Paris, 1969).

[4] Isidore Fleischer, "On 'Subcompactness and the Baire category theorem"", Nederl. Akad. Wetensch. Proc. Ser. A 82 = Indag. Math. 41 (1979), 9-11.

[5] Isidore Fleischer and Gonzalo E. Reyes, The N-Baire property (CRM Preprint, 425. Centre de Recherches Mathematiques et Department de Mathematiques, Montreal, 1974).

[6] G. Gierz, K.H. Hofmann, K. Keimel, J.D. Lawson, M. Mislove, D.S. Scott, A compendium of continuous Lattices (SpringerVerlag, Berlin, Heidelberg, New York, to appear).

[7] J. de Groot, "Subcompactness and the Baire category theorem", Nederl. Akad. Wetensch. Proc. Ser. A 66 = Indag. Math. 25 (1963), 761-767.

[8] Rudolf -E. Hoffmann, "Charakterisierung nüchterner Räume", Manuscripta Math. 15 (1975), 185-191.

[9] Karl Heinrich Hofmann, "Continuous lattices, topology and topological algebra", Topology Proc. 2 (1977), 179-212.

[10] Karl H. Hofmann and Jimmie D. Lawson, "Irreducibility and generation in continuous lattices", Semigroup Forum $13(1976 / 77), 307-353$. 
[11] Karl H. Hofmann and Jimmie D. Lawson, "The spectral theory of distributive continuous lattices", Trans. Amer. Math. Soc. 246 (1978), 285-310.

[12] John R. Isbell, "Function spaces and adjoints", Math. Scand. 36 (1975), 317-339.

[13] J.D. Lawson, "Joint continuity in semitopological semigroups", IZZinois J. Math. 18 (1974), 275-285.

[14] Jimmie D. Lawson, "Additional notes on continuity in semitopological semigroups", Semigroup Forum 12 (1976), 265-280.

[15] 1. Namioka, "Separate continuity and joint continuity", Pacific $J$. Math. 51 (1974), 515-531.

[16] Dana Scott, "Continuous lattices", Toposes, algebraic geometry and Zogic, 97-136 (Lecture Notes in Mathematics, 274. SpringerVerlag, Berlin, Heidelberg, New York, 1972).

[17] A.S. Ward, Problem, p. 352 of Proceedings of the Intermational Symposium on Topology and its Applications, Herceg-Novi, 1968, Yugoslavia [Tруды Mendyнародного Сuмnозия nо топологии u ee Применениях, Херцег-Нови, 1968, Ю-ославия] (Savez Drustava Matematicara, Fizicara I Astronoma, Belgrade, 1969).

Department of Mathematics,

Tulane University,

New Orleans,

Louisiana 70118 ,

USA . 